\title{
Coexisting hypothyroidism - Does it aggravate PCOS?
}

\author{
Arun Mathew Chacko', Geetha ${ }^{2, *}$, Beena Kumari ${ }^{3}$, Deepti G. N. ${ }^{4}$, Sumina Cherian ${ }^{5}$ \\ ${ }^{\mathbf{1 , 4 , 5}}$ Assistant Professor, ${ }^{2}$ Professor, ${ }^{\mathbf{3}}$ Additional Professor, $\mathbf{1 , 2 , 4 , 5}$ Dept. of Biochemistry, 3Dept. Of Obstetrics and Gynecology, \\ ${ }^{1}$ KMCT Medical College, Calicut, Kerala, ${ }^{2,4,5}$ Pushpagiri Institute of Medical Sciences and Research Centre Thiruvalla, Kerala, \\ ${ }^{3}$ Government Medical College, Kottayam, Kerala, India
}

Received: $05^{\text {th }}$ December, 2017

*Corresponding Author:

Email: rnsharmas_revathy@hotmail.com

Accepted: $08^{\text {th }}$ March, 2018

\begin{abstract}
Introduction: The goal of the present study was to find out the effect of coexisting hypothyroidism on the biochemical and anthropometric measurements in PCOS patients.

Materials and Methods: The study consisted of 90 subjects already diagnosed as PCOS and thirty age matched controls .Biochemical parameters like fasting blood sugar and lipid profile and the anthropometric measurements like BMI and waist circumference were compared between the two groups. The 90 PCOS subjects were further divided in to two depending on their thyroid status as euthyroid and hypothyroid and the biochemical and anthropometric parameters were compared between these two groups.

Result: In our study there was no statistically significant difference in the biochemical parameters between the PCOS subjects and the control group but the anthropometric parameters BMI and waist circumference were markedly increased in PCOS subjects as compared to the control group. The fasting blood sugar, total cholesterol (TC), LDL cholesterol and TG (triglyceride) levels were increased in hypothyroid PCOS subjects as compared to the euthyroid PCOS subjects. The waist circumference was also markedly increased in hypothyroid PCOS subjects as compared to the euthyroid group.

Conclusion: Fasting blood sugar, Total cholesterol, LDL cholesterol and triglycerides were significantly increased in hypothyroid PCOS patients as compared to euthyroid PCOS patients. This may increase the risk of developing diabetes and cardiovascular events in PCOS patients suffering form hypothyroidism
\end{abstract}

Keywords: Polycystic ovary syndrome, Subclinical hypothyroidism, Lipid profile, Body mass index, Waist circumference.

\section{Introduction}

Thyroid disorders and polycystic ovary syndrome (PCOS) are two of the most common endocrine disorders in the general population. PCOS is a heterogeneous androgen excess disorder of multifactorial aetiology whose diagnosis is based on anthropometric, biochemical and radiological abnormalities. It is characterized by irregular menstruation (IM), obesity, hyperandrogenism (HA) and polycystic ovary (PCO). It is also associated with increased metabolic and cardiovascular risk factors. ${ }^{1}$ Elevated luteinizing hormone (LH) and insulin resistance are two of the most common aberrations seen in PCOS. Insulin being an anabolic hormone, the hyperinsulinemia associated with insulin resistance leads to obesity and increased waist circumference. It is not clear whether hypothyroidism alters the metabolic $\&$ anthropometric parameters of these patients. ${ }^{2}$

Dysfunction and anatomic abnormalities of the thyroid is associated with alteration in a number of metabolic processes. Subclinical thyroid dysfunction can lead to noticeable changes in ovulation and endometrial receptivity. Severe hypothyroidism may be associated with anovulation and diminished libido. Primary ovarian failure can also be seen in patients with autoimmune thyroid disorders. Rarely, in primary hypothyroidism, secondary depression of pituitary function may lead to ovarian atrophy and amenorrhoea.
The role of abdominal obesity as a contributing factor in the development of PCOS is widely accepted and may be responsible for IR and associated hyperinsulinemia in women with PCOS..$^{3-5}$ Presence of obesity can amplify the consequences of PCOS. Around $30-50 \%$ of PCOS patients are obese with tendency to have increased abdominal obesity ${ }^{6}$ Waist circumference (WC) is one of the common proxy measure of visceral adipose tissue and is strongly associated with metabolic risk factors. In the present study, we have evaluated the thyroid status, other biochemical and anthropometric characteristics of PCOS subjects and compared them with healthy controls. We also made an attempt to compare the biochemical \& anthropometric parameters in hypothyroid \& euthyroid PCOS patients.

\section{Materials and Methods}

This cross sectional study consists of ninety subjects with already diagnosed PCOS between the age group 17 to $35 \&$ thirty age matched healthy controls. Patients on oral contraceptive pills, statins \& other medications that alter thyroid functions, pregnant women, patients with liver disorders, renal disorders, congestive cardiac failure or any thyroid disorders and acutely ill patients were excluded from the study. Study was conducted after obtaining approval of the Institutional Review Board (IRB No: - 24/2014). 
Anthropometric measurements like weight, height, waist circumference were recorded. BMI was calculated using the formula Weight in $\mathrm{Kg}$ / height in $\mathrm{m}^{2}$. Blood pressure were recorded for all the subjects. Biochemical parameters like TSH, Free T4, Free T3, Total cholesterol, HDL, LDL and triglycerides were estimated in Fasting serum sample in all the subjects. TSH, FT4, FT3 were analysed in Cobas e 411 analyser based on Electrochemiluminescense Immunoassay (ECLIA). Other serum parameters were analysed in Beckman Coulter AU 480. All the results were tabulated and statistically analysed using SPSS software. $\mathrm{P}$ value $<0.05$ is considered as statistically significant.

\section{Result}

Ninety subjects with already diagnosed PCOS between the age group 17 to $35 \&$ thirty age matched healthy controls were selected and analysed for the present study. Of the 90 cases, 22 (24\%) were hypothyroid \& $68(76 \%)$ were euthyroid. There is no cases of hyperthyroidism in our present study. Thyroid function was evaluated using TSH, Free T3 \& Free T4. The values are expressed as Mean \pm SD. The mean TSH of subjects with PCOS is $3.6 \pm 2.82 \mu \mathrm{IU} / \mathrm{ml}$ and subjects without PCOS is $3.15 \pm 2.05 \mu \mathrm{IU} / \mathrm{ml}$. Normal range for TSH is $0.27-4.2 \mu \mathrm{IU} / \mathrm{ml}$. A high serum TSH level $4.2-10 \mu \mathrm{IU} / \mathrm{ml}$ and normal FT3 \& FT4 levels are required for the diagnosis of subclinical hypothyroidism. Subclinical hypothyroidism refers to increased serum TSH levels in the setting of normal FT3 \& FT4 estimates. Patients with high TSH $>10 \mu$ $\mathrm{IU} / \mathrm{ml}$ and low FT3 \& FT4 levels are classified as being overtly hypothyroid. Patients with normal TSH, FT3 \& FT4 are considered as euthyroid.

In the present study, the mean FT3 values of PCOS subjects and control subjects were $3.56 \pm 0.59 \& 3.39 \pm$ 0.53 respectively. The mean FT4 values of PCOS subjects and control subjects were $1.14 \pm 0.31$ and $1.121 \pm 0.36$ respectively. The mean TC values of PCOS $\&$ control subjects were $184.9 \pm 29.9 \& 176.6 \pm 24.27$ respectively. The mean triglyceride levels of PCOS \& control subjects were $106.3 \pm 32.7 \& 94.73 \pm 31.49$ respectively. The mean HDL levels of PCOS \& control subjects were $52.06 \pm 9.95 \& 52.87 \pm 8.94$ respectively. The mean LDL levels of PCOS \& control subjects were $111.59 \pm 28.44 \& 104.87 \pm 23.97$ respectively. The mean FBS levels of PCOS \& control subjects were $94.02 \pm 11.94 \& 91.83 \pm 8.54$ respectively. The triglyceride values were higher in PCOS subjects compared to controls but it is not statistically significant.

The mean BMI of PCOS \& control subjects were $24.42 \pm 1.99 \& 22.53 \pm 2.53$ respectively. The mean waist circumference of PCOS \& control subjects were $33.36 \pm 3.61 \& 30.63 \pm 2.34$ respectively. BMI and waist circumference were significantly increased in PCOS subjects compared to control subjects (P value < $0.001)$

Table 1: Comparison of Biochemical parameters in PCOS subjects and Controls

\begin{tabular}{|l|c|c|c|}
\hline \multicolumn{1}{|c|}{ Parameters } & Cases & Controls & P value \\
\hline TSH $(\mu \mathrm{IU} / \mathrm{ml})$ & $3.61 \pm 2.82$ & $3.15 \pm 2.05$ & 0.34 \\
\hline FT3 $(\mathrm{pg} / \mathrm{ml})$ & $3.56 \pm 0.59$ & $3.39 \pm 0.53$ & 0.16 \\
\hline FT4 $(\mathrm{ng} / \mathrm{dl})$ & $1.14 \pm 0.31$ & $1.121 \pm 0.36$ & 0.8 \\
\hline TC $(\mathrm{mg} / \mathrm{dl})$ & $184.9 \pm 29.9$ & $176.63 \pm 24.27$ & 0.13 \\
\hline Triglyceride $(\mathrm{mg} / \mathrm{dl})$ & $106.3 \pm 32.7$ & $94.73 \pm 31.49$ & 0.09 \\
\hline HDL $(\mathrm{mg} / \mathrm{dl})$ & $52.06 \pm 9.95$ & $52.87 \pm 8.94$ & 0.67 \\
\hline LDL $(\mathrm{mg} / \mathrm{dl})$ & $111.59 \pm 28.44$ & $104.87 \pm 23.97$ & 0.21 \\
\hline FBS $(\mathrm{mg} / \mathrm{dl})$ & $94.02 \pm 11.94$ & $91.83 \pm 8.54$ & 0.27 \\
\hline
\end{tabular}

Table 2: Comparison of Anthropometric parameters in PCOS subjects and Controls

\begin{tabular}{|l|c|c|c|}
\hline \multicolumn{1}{|c|}{ Parameters } & Cases & Controls & P value \\
\hline BMI $\left(\mathrm{Kg} / \mathrm{M}^{2)}\right.$ & $24.42 \pm 1.989$ & $22.53 \pm 2.53$ & $<0.001$ \\
\hline $\begin{array}{l}\text { Waist } \\
\text { Circumference }(\mathrm{cms})\end{array}$ & $33.36 \pm 3.61$ & $30.63 \pm 2.34$ & $<0.0001$ \\
\hline
\end{tabular}

Of the 90 PCOS patient $\mathrm{s}$ there 22 were hypothyroid and 68 were euthyroid. Biochemical parameters and anthropometric measurements were compared between these 2 groups.

The mean TSH of PCOS patients with hypothyroidism was $7.84 \pm 2.45$ and the mean value of TSH in euthyroid PCOS patients was $2.24 \pm 0.95$. This mean TSH value is significantly higher in hypothyroid
PCOS patients than in euthyroid PCOS patients ( $p$ value $<0.0001$ ). The mean FreeT3 of PCOS patients with hypothyroidism was $3.36 \pm 0.76$ and the mean value of Free T3 in euthyroid PCOS patients was $3.62 \pm 0.51$. The mean Free T4 of PCOS patients with hypothyroidism was $1.08 \pm 0.46$ and the mean value of FreeT4 in euthyroid PCOS patients was $1.16 \pm 0.25$ 
The mean Fasting blood sugar of PCOS patients with hypothyroidism was $104.55 \pm 14.3$ and the mean value of FBS in euthyroid PCOS patients was $90.62 \pm$ 8.79. The mean FBS value is significantly higher in hypothyroid PCOS patients than in euthyroid PCOS patients ( $\mathrm{p}$ value - 0.0002).

The mean total cholesterol of PCOS patients with hypothyroidism was $210.95 \pm 34.71$ and the mean value of total cholesterol in euthyroid PCOS patients was $176.5 \pm 22.76$. The mean total cholesterol value is significantly higher in hypothyroid PCOS patients than in euthyroid PCOS patients ( $p$ value -0.0002). The mean HDL cholesterol of PCOS patients with hypothyroidism was $49.05 \pm 10.34$ and the mean value of HDL cholesterol in euthyroid PCOS patients was $53.03 \pm 9.7)$. The mean LDL cholesterol of PCOS patients with hypothyroidism was $136.73 \pm 32.98$ and the mean value of LDL cholesterol in euthyroid PCOS patients was $103.46 \pm 21$.43.The mean LDL cholesterol levels was significantly higher in hypothyroid PCOS patients as compared to euthyroid PCOS patients ( $\mathrm{p}$ value 0.0001 ). The mean Triglyceride of PCOS patients with hypothyroidism was $126.23 \pm 37.32$ and the mean value of triglyceride in euthyroid PCOS patients was $99.85 \pm 28.43$. Triglyceride levels were higher in hypothyroid patients with PCOS compared with euthyroid patients with PCOS ( $p$ value 0.0049 ).

The mean BMI of PCOS patients with hypothyroidism was $24.91 \pm 2.45$ and the mean value of BMI in euthyroid PCOS patients was $24.26 \pm 1.81$. The mean Waist circumference of PCOS patients with hypothyroidism was $36.41 \pm 3.5$ and the mean value of Waist circumference in euthyroid PCOS patients was $32.39 \pm 3.06$. The mean waist circumference of hypothyroid PCOS patients was significantly higher as compared to euthyroid PCOS patients ( $p$ value< $0.0001)$

Table 3: Comparison of Biochemical parameters between hypothyroid and euthyroid PCOS patients

\begin{tabular}{|l|l|l|l|}
\hline \multicolumn{1}{|c|}{ Parameters } & $\begin{array}{c}\text { PCOS hypothyroid } \\
\text { patients }\end{array}$ & $\begin{array}{c}\text { PCOS Euthyroid } \\
\text { patients }\end{array}$ & P value \\
\hline $\mathrm{TSH}(\mu \mathrm{IU} / \mathrm{ml})$ & $7.84 \pm 2.45$ & $2.24 \pm 0.95$ & $<0.0001$ \\
\hline Free $\mathrm{T} 3(\mathrm{pg} / \mathrm{ml})$ & $3.36 \pm 0.76$ & $3.62 \pm 0.51$ & 0.154 \\
\hline Free $\mathrm{T} 4(\mathrm{ng} / \mathrm{dl})$ & $1.08 \pm 0.46$ & $1.16 \pm 0.25$ & 0.462 \\
\hline FBS $(\mathrm{mg} / \mathrm{dl})$ & $104.55 \pm 14.27$ & $90.62 \pm 8.79$ & 0.0002 \\
\hline $\mathrm{HDL}(\mathrm{mg} / \mathrm{dl})$ & $49.05 \pm 10.34$ & $53.03 \pm 9.7$ & 0.12 \\
\hline $\mathrm{LDL}(\mathrm{mg} / \mathrm{dl})$ & $136.73 \pm 32.98$ & $103.46 \pm 21.43$ & 0.0001 \\
\hline $\mathrm{TG}(\mathrm{mg} / \mathrm{dl})$ & $126.23 \pm 37.32$ & $99.85 \pm 28.43$ & 0.0049 \\
\hline $\mathrm{TC}(\mathrm{mg} / \mathrm{dl})$ & $210.95 \pm 34.71$ & $176.5 \pm 22.76$ & 0.0002 \\
\hline
\end{tabular}

Table 4: Comparison of anthropometric measurements between hypothyroid and euthyroid PCOS patients

\begin{tabular}{|l|l|l|l|}
\hline \multicolumn{1}{|c|}{ Measurements } & $\begin{array}{c}\text { PCOS hypothyroid } \\
\text { patients }\end{array}$ & $\begin{array}{c}\text { PCOS Euthyroid } \\
\text { patients }\end{array}$ & P value \\
\hline $\mathrm{BMI}\left(\mathrm{Kg} / \mathrm{M}^{2}\right)$ & $24.91 \pm 2.45$ & $24.26 \pm 1.81$ & 0.264 \\
\hline Waist circumference $(\mathrm{cms})$ & $36.41 \pm 3.54$ & $32.37 \pm 3.06$ & $<0.0001$ \\
\hline
\end{tabular}

On correlating thyroid parameters with different biochemical parameters and anthropometric measurements in PCOS patients, there was a positive correlation between TSH and TC (P value - <0.0001), TSH and LDL (P value $-<0.0001)$, TSH and TG $(\mathrm{P}$ value -0.0005$)$. We also found a positive correlation between TSH and Waist circumference ( $\mathrm{P}$ value $<0.0001$ ). There was significant negative correlation of Free T4 with lipid parameters like TC, LDL and TG with $\mathrm{p}$ values $<0.05$.

\section{Discussion}

Various studies have shown that hypothyroidism is more common in women with poycystic ovarian disease (PCOS) as compared to the general population. But in our present study, there was no significant increase in the number of hypothyroid patients in PCOS group compared with the control subjects. In our study
$24 \%$ of PCOS subjects had hypothyroidism and there was no cases of hyperthyroidism. This shows that thyroid disorders which is more common in PCOS subjects is hypothyroidism. A study conducted by Sinha et al. have also reported $22.5 \%$ subclinical hypothyroidism and $2.5 \%$ clinical hypothyroidism in PCOS subjects which are very close to our study. ${ }^{1}$ Hypothyroid women have decreased clearance of androstenedione and estrone and exhibit an increase in peripheral aromatization of androstenedione to estrogen. Plasma level of Sex hormone binding globulin is decreased which results in increase in their unbound fraction and the levels of unbound fraction is normalised when euthyroidism is achieved. Since treatment of the hypothyroid state may favourably influence the metabolic alteration in PCOS, it is advisable to do a thyroid profile in all PCOS patients.

Women with polycystic ovary syndrome are more susceptible to coronary artery disease. This may be 
partly due to the alteration of lipid profile in these patients. In this study, there was no significant variation in lipid profile parameters between PCOS subjects and normal subjects. This may be due to the younger age group of the study subjects (17-35 years)

While evaluating the anthropometric parameters both BMI and waist circumference were significantly increased in PCOS patients as compared to the control group. Previous studies also showed that obesity is a common finding in women with PCOS and between $40-80 \%$ of women with this condition are reported to be overweight or obese. Some studies have shown that hyperandrogenism associated with PCOS causes central obesity as indicated by the increased waist circumference independant of the body mass index. Study by Escobar et al showed that systemic hyperinsulinism plays a major role in the development of the hyperandrogenism which alters the $\beta$-cell function. ${ }^{8-10}$

We subdivided the PCOS subjects based on their thyroid status as hypothyroid and euthyroid PCOS patients and compared the biochemical and anthropometric parameters between these groups.

The total cholesterol, LDL cholesterol and serum triglycerides were significantly increased in hypothyroid PCOS patients as compared to euthyroid PCOS patients. ${ }^{12-14}$ This clearly shows that hypothyroidism can cause metabolic derangements in PCOS subjects which can have an adverse effect. It is a well established fact that serum cholesterol levels are increased in patients with hypothyroidism and treatment of hypothyroidism will lead to lowering of serum cholesterol levels. The HUNT study by Bjorn $\mathrm{O}^{\circ}$ svold in Norway showed that the total serum cholesterol, LDL cholesterol, and triglycerides were increased and HDL-C is decreased consistently with increase in TSH levels. In the present study there was no statistically significant variation in the HDL levels of hypothyroid and euthyroid PCOS patients. The high total serum cholesterol and LDL cholesterol may be due to fewer LDL receptors on the cell surface. ${ }^{15}$ The increase in serum triglyceride level is due to decreased activity of lipoprotein lipase ${ }^{16}$ and also due to decreased LDL receptors. Insulin resistance leads to increased production of VLDL which also increases LDL and triglycerides.

The mean fasting blood sugar of hypothyroid PCOS patients was significantly higher as compared to euthyroid PCOS patients. A study conducted by Ganie et.al showed that hypothyroidism is associated with increased insulin resistance and it also exerts a direct effects on insulin secretion and clearance which is supported by our present study Insuin resitance is increased in hypothyroidism which leads to weight gain. ${ }^{17,18}$

In normal individuals serum cholesterol levels show an inverse relationship with serum free T4 levels .Moreover, low normal FT4 levels were significantly associated with increased insulin resistance. These findings are consistent with an increased cardiovascular risk in subjects with low normal thyroid function. ${ }^{19}$

We also studied the correlation between the thyroid hormone levels and the various biochemical and anthropometric parameters in PCOS subjects.

There was a positive correlation between TSH and TC (P value - $<0.0001)$, TSH and LDL ( $\mathrm{P}$ value $<0.0001$ ), TSH and TG (P value -0.0005). We also found a positive correlation between TSH and Waist circumference $(\mathrm{P}$ value $-<0.0001)$. A study conducted by Bates et.al showed that the greatest health implications of PCOS are associated with excess weight and abdominal circumference ${ }^{20}$ because a greater visceral adiposity is associated with a greater IR. There was significant negative correlation of Free T4 with lipid parameters like TC, LDL and TG with $\mathrm{p}$ values < 0.05 .

Even though there was no significant difference in lipid profile in PCOS subjects as compared to the control group there was a statistically significant positive correlation between the TSH values and TC, LDL cholesterol and TG levels like the study by Kalra et al. who compared lipid profile between thyroid parameters in PCOS groups. High levels are associated with hypothyroidism and lowering of thyroid hormone levels results in elevation of TC, LDL cholesterol and TG in these patients. ${ }^{21}$

\section{Conclusion}

PCOS is associated with increased cardiovascular and metabolic risk factors. According to the present study coexisting hypothyroidism causes an increase in the fasting blood glucose level and adversely affects the lipid profile. This mayincrease the cardiovascular and metabolic risk factors in these patients. All patients with PCOS should be routinely screened for thyroid dysfunction and treatment of hypothyroidism will have long term health benefits.

\section{References}

1. Sinha U, Sinharay K, Saha S, Longkumer TA, Baul SN, $\mathrm{Pal}$ SK. Thyroid disorders in polycystic ovarian syndrome subjects:A tertiary hospital based cross-sectional study from EasternIndia. Indian J Endocrinol Metab. 2013;17(2):304-9.

2. European Journal of Endocrinology March 12017176 R159-R166.

3. Balen A.The current understanding of polycystic ovary syndrome The Obstetrician \& Gynaecologist ,6,66-74.

4. Fauser BC, Tarlatzis BC, Rebar RW, Legro RS, Balen $\mathrm{AH}$, Lobo R et al. Consensus on women's health aspects of polycystic ovary syndrome: the Amsterdam ESHRE/ASRM-Sponsored $3^{\text {rd }}$ PCOS consensus Workshop Group Fertil Steril 2012 Jan;28-38 e25.

5. Padubiri VG, Daftary SN, Shaws's Textbook of Gynaecology-15 $5^{\text {th }}$ edition2011; Chapter4, Paediatric Gynaecology, Adolescent Problems and Puberty:110-30. 
6. Escobar-Morreale HF, San Millan JL, Abdominal adiposity and the polycystic ovary syndrome Trends Endocrinol Metab.2007;18(7) 266-72.

7. Liang SJ, Liou TH, Lin HW, et al. Obesity is the predominant predictor of impaired glucose tolerance and metabolic disturbance in polycystic ovary syndrome. Acta Obstet Gynecol Scand, 2012.Oct;91(10):1167-72.

8. Bates GW, Legro RS. Long-term management of Polycystic Ovarian Syndrome (PCOS) Mol Cel Endocrinol. 2013;373(1-2):91-7.

9. Carmina E, Bucchieri S, Esposito A, et al. Abdominal fat quantity and distribution in women with polycystic ovary syndrome and extent of its relation to insulin resistance. J Clin Endocrinol Metab. 2007;92(7):2500-5.

10. Cascella T, Palomba S, De Sio I, et al. Visceral fat is associated with cardiovascular risk in women with polycystic ovary syndrome. Hum Reprod. 2008;23(1):153-9.

11. Lord J, Thomas R, Fox B, Acharya U, Wilkin T. The central issue? Visceral fat mass is a good marker of insulin resistance and metabolic disturbance in women with polycystic ovary syndrome. BJOG. 2006;113(10):1203-9.

12. Ganie MA, Laway BA, Wani TA, et al. Association of subclinical hypothyroidism and phenotype, insulin resistance and lipid parameters in young women with polycystic ovary syndrome. Fertil Steril. 2011 May; 95(6)2039-43.

13. Roos A, Bakker SJ, Links TP, Gans RO, Wolffenbuttel $\mathrm{BH}$. Thyroid function is associated with components of metabolic syndrome in euthyroid subjects. J Clin Endocrinol Metab. 2007;92:491-6.

14. Celik C, Abali R, Tasdemir N, Guzel S, Yuksel A, Aksu $\mathrm{E}$, et al. Is subclinical hypothyroidism contributing dyslipidemia and insulin resistance in women with polycystic ovary syndrome? Gynecol Endocrinol. 2012;28:615-8.

15. Rabelo AM, Vick MR. Association between the PCOS and the metabolic syndrome in Puerto rico. PR Health Sci J2005;24(3):203-6.

16. Reaven GM, Banting lecture 1988.Role of insulin resistance in human disease. Diabetes37:1595-607.

17. Celik C, Abali R, Tasdemir N, Guzel S, Yuksel A, Aksu $\mathrm{E}$, et al. Is subclinical hypothyroidism contributing dyslipidemia and insulin resistance in women with polycystic ovary syndrome? Gynecol Endocrinol. 2012;28(8):615-18

18. Enzevaei S, Salehpour M, Tohidi, Saharkhiz N Subclinical hypothyroidism and insulin resistance in polycystic ovary syndrome: is there a relationship? Iranian Journal of Reproductive Medicine, vol. 12, no. 7, pp. 481-486,2014.

19. Muscogiuri G, Sorice GP, Mezza T, Prioletta A, Lassandro AP, Pirronti T, et al. High-normal TSH values in obesity: Is it insulin resistance or adipose tissue's guilt? Obesity (Silver Spring) 2013;21:101-6.

20. Bates GW, Legro RS. Long-term management of Polycystic Ovarian Syndrome (PCOS) Mol Cel Endocrinol. 2013;373(1-2):91-7.

21. Kalra A, Nair S, Rai L. Association of obesity and insulin resistance with dyslipidemia in Indian women with polycystic ovarian syndrome. Indian J Med Sci. 2006;60(11):447. 